\title{
Contractile and electrical properties of human motor units in neuropathies and motor neurone disease
}

\author{
H. S. MILNER-BROWN, R. B. STEIN, ${ }^{1}$ AND R. G. LEE \\ From the Department of Physiology, University of Alberta, Edmonton, \\ and Department of Medicine (Neurology), University of Toronto \\ (Toronto Western Hospital), Toronto, Canada
}

SYNOPSIS The contractile and electrical properties of motor units in the first dorsal interosseous muscle of the hand have been studied in 26 patients with ulnar neuropathies and motor neurone $\overrightarrow{0}$ disease (amyotrophic lateral sclerosis). Among patients with unilateral pressure or entrapment ulnar $\overrightarrow{\vec{\omega}}$ neuropathies, there was a tendency for the twitch tensions for single motor units to be smaller, while $\stackrel{\sigma}{S}$ the surface EMG amplitudes were generally larger in the affected hands. Very large EMG amplitudes $\overline{\bar{z}}$ but normal size twitch tensions were observed among the motor neurone disease patients, indicating? that, in general, motor units enlarged by sprouting are less efficient contractile units than units of normal physiological size.

The ulnar nerve may be damaged as a result of localized pressure or entrapment at various sites along its course. The nerve is most vulnerable at the elbow, where it travels in a shallow groove behind the medial epicondyle. Here it may be damaged due to external pressure (either a single episode such as occurs during a period of unconsciousness or multiple episodes of pressure such as in the office worker who leans on his elbow). The nerve may also become entrapped and compressed in fibrous tissue in the ulnar groove, or it may be damaged as a result of a congenital or acquired deformity at the elbow, in which case repeated flexion and extension of the joint traumatizes the nerve (the so-called tardy ulnar palsy). Anterior transposition is frequently performed, but completely normal function is seldom regained (Seddon, 1972). The prognosis after surgery depends largely on the degree of nerve involvement and Payan (1970) contends that the operation is being performed more often than necessary.

Previous electrophysiological studies of motor neurone disease have indicated the following changes in motor unit properties (Erminio et al., 1959; Buchthal and Olsen, 1970): (1) increased

1 Address for correspondence: Dr. R. B. Stein, Department of Physiology, University of Alberta, Edmonton, Canada. amplitude of the individual motor unit action potential; (2) increased amplitude of the inter- $c$ ference pattern during strong voluntary effor:⿱乛龰⿱中⿰㇀丶㇀ (3) $30 \%$ or more increase in the mean duration of $\vec{\omega}$ the motor unit action potential; and (4) $80 \%$ 국 more increase in the territorial area of the motor unit. Very little, however, is known concerning the twitch tensions and other mechanical properties of these altered motor units.

A detailed electrophysiological study has been $\frac{2}{\triangleright}$ made of (a) 14 patients with unilateral and four $\overrightarrow{\vec{A}}$ bilateral pressure or entrapment neuropathies $\overline{3}$ affecting the ulnar nerve; and (b) eight patients? with motor neurone disease (amyotrophic lateralo sclerosis). In these two groups there is a possibility that relatively normal motoneurones can sprout new collaterals and innervate more muscle fibres $\frac{\sigma}{3}$ to compensate for the loss of other motoneurones (Coërs and Woolf, 1959). The extent of functional compensation resulting from enlarged motor units is unknown. In addition, there could be different effects due to the site of the lesion which will most severely affect nerve conduction (group a) or the properties of the cell bodyo (group b).

\section{METHODS}

The method for measuring the contractile propertieso 
a

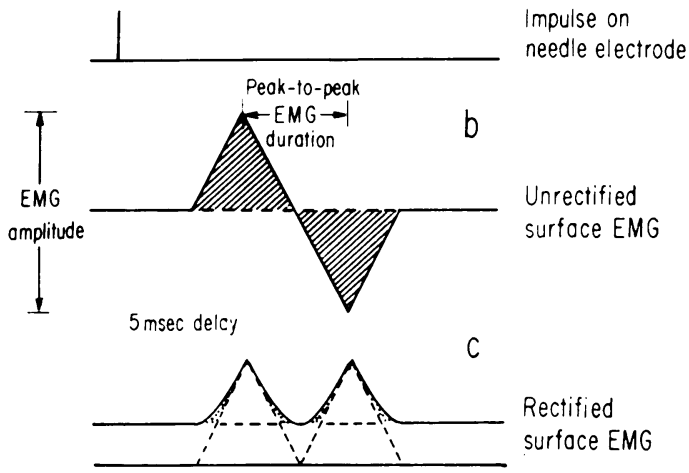

d

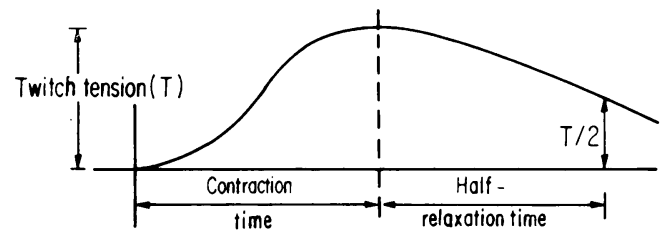

FIG. 1. Schematic representation of the averaged twitch tension, with the parameters of the twitch which are routinely measured (d) and a test for synchronization of motor units using the unrectified and rectified surface EMGs $(\mathrm{a}-\mathrm{c})$. If a laboratory computer is triggered by impulses from a single unit (a) recorded by a needle electrode, a waveform (b) is obtained by directly averaging the surface EMG. When impulses in different motor units are generated independently (no synchronization), this average will simply represent the average voltage generated by the single unit which is recorded by the surface electrodes (the area indicated by diagonal lines in $\mathrm{b}$ ). If averaging is done after rectification (c), there will be a net contribution to the average of (1) the waveform in $\mathrm{b}$ after rectification, (2) the ongoing activity of other units, and (3) a slight degree of summation between these two (dotted area). However, if the discharge of several units tends to be grouped (synchronization) a broader and larger increase in the rectified surface EMG will be observed than can be accounted for by (1) to (3) above.

of single motor units during isometric voluntary contractions while simultaneously recording the electrical activity using surface and needle electrodes has been described in the previous paper and in more detail in Milner-Brown et al. (1973a), so only the method of measuring synchronization of motor units will be discussed below.
SYNCHRONIZATION The presence of synchronization was evaluated quantitatively using the unrectified and rectified surface electromyogram (EMG) records. The surface EMG was passed through a full-wave rectifier, and then averaged as a function of time before or after the occurrence of impulses from a motor unit recorded by a needle electrode. The effect of this process is shown schematically in Fig. 1. The time of occurrence of impulses recorded by the needle electrode is indicated by a vertical line in Fig. 1a. Associated with these impulses will be some waveform of an unrectified average (indicated schematically by a triangular waveform in Fig. 1b). The surface EMG was electronically delayed $5 \mathrm{~ms}$ before recording. Impulses from other units which are not tightly synchronized will be 'averaged' out because there will be an equal probability that they will give a positive or a negative contribution on successive sweeps. (Because of the a.c. recording, the time average of these EMG waveforms must be zero.) From Fig. 1, a synchronization ratio is given by the dotted area in Fig. 1c over the area indicated by diagonal lines in Fig. 1b. The theoretical basis of this argument is explained in Milner-Brown et al. (1973a). Without synchronization, the degree of summation using the waveform of Fig. $1 \mathrm{~b}$ is under $10 \%$ (Milner-Brown et al., 1973a). The expected summation depends on the actual waveforms recorded and a summation of $20 \%$ was considered as a reasonable lower limit for synchronized activity. In general the synchronization ratios obtained might have been under-estimated, since the maximum delay available for the surface EMG was $5 \mathrm{~ms}$. Synchronized activity recorded on the surface more than $5 \mathrm{~ms}$ before the impulses from a motor unit was therefore neglected.

STATISTICAL TREATMENT The significance of a difference between the distributions of the same parameters in the normal and affected hands was calculated using the Kolmogorov-Smirnov $\lambda$ test (Fisz, 1963).

\section{RESULTS}

PRESSURE OR ENTRAPMENT ULNAR NEUROPATHIES Twitch tension Among the unilateral ulnar neuropathy patients, there was a tendency for the twitch tensions to be smaller in the affected hand. The overall means were $5 \cdot 94 \pm 1 \cdot 3 \mathrm{~g}$ (mean $\pm \mathrm{SE}$ of the mean) for motor units in the normal hands and $3.66 \pm 0.69 \mathrm{~g}$ for motor units in the abnormal hands of 14 patients. There was also a general tendency for the contraction and half-relaxation times to be longer in the affected hands as 
illustrated in the histogram in Fig. 2. The mean values were $72 \pm 20 \mathrm{~ms}$ (mean \pm SD) for the contraction times and $70 \pm 19 \mathrm{~ms}$ for the halfrelaxation times of affected hands. The corresponding values in normal hands were $61 \pm$ $17 \mathrm{~ms}$ for the contraction times and $52 \pm$ $14 \mathrm{~ms}$ for the half-relaxation times. The distributions were significantly different $(P<0.01$ for both parameters).

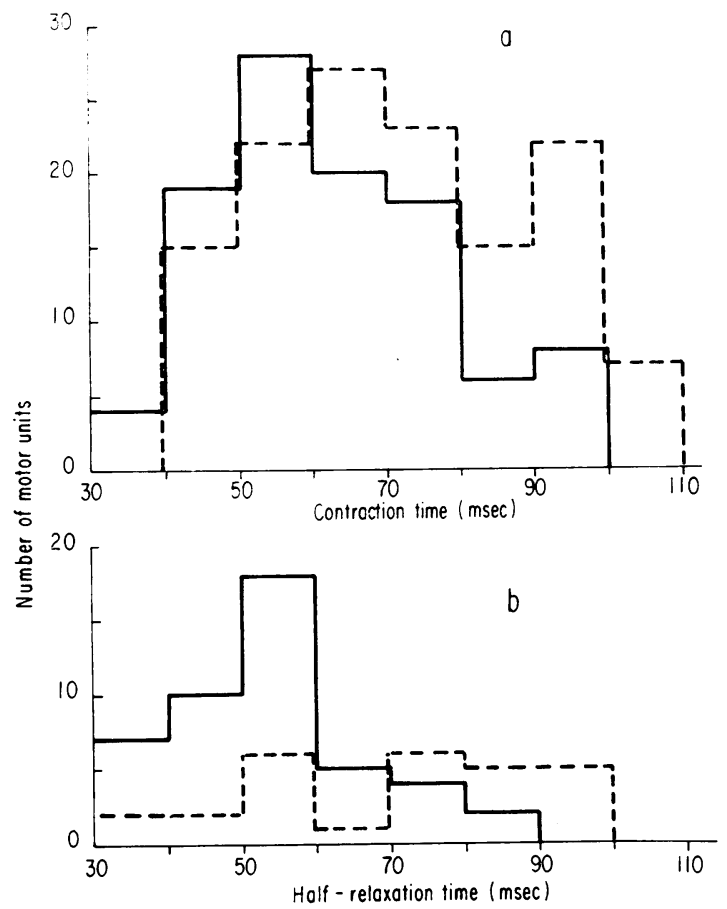

FIG. 2. a Contraction times of twitches from motor units in normal hands (-) and affected hands (---) of 14 patients with unilateral ulnar neuropathies. b Half-relaxation times of twitches from motor units in normal hands $(-)$ and affected hands (--) of 14 patients with unilateral ulnar neuropathies. The distributions were significantly different $(P<0.01$ for both parameters).

EMG amplitudes The EMG amplitudes were generally larger $(2 \times$ or more) in the affected hand. These results suggest the possibility of sprouting of healthy motoneurones to reinnervate the muscle fibres that had become denervated (Wohlfart, 1957, 1958; Coërs and Woolf, 1959;

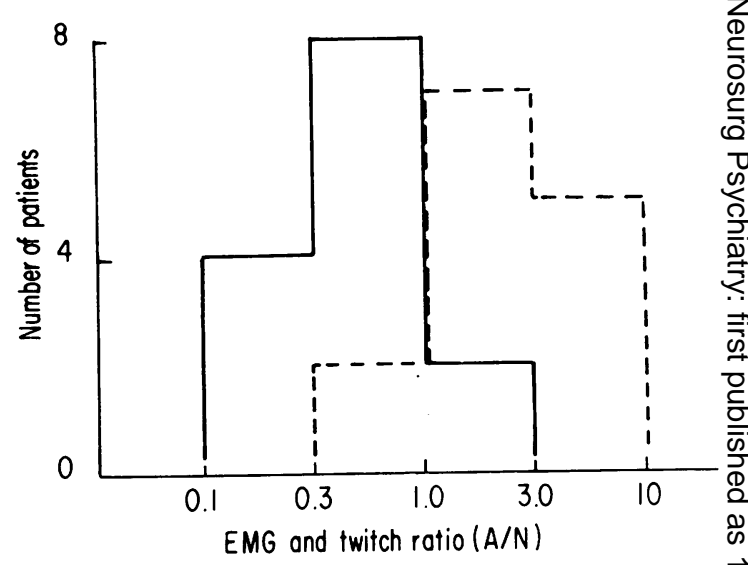

FIG. 3. Mean twitch tension ratios $(-)$ and mean EMG amplitude ratios (---) of affected (A) and $\overrightarrow{\vec{\omega}}$ normal $(\mathrm{N})$ hands from 14 patients with unilateral ulnar neuropathies. The distributions of the ratios were significantly different $(P<0 \cdot 01)$.

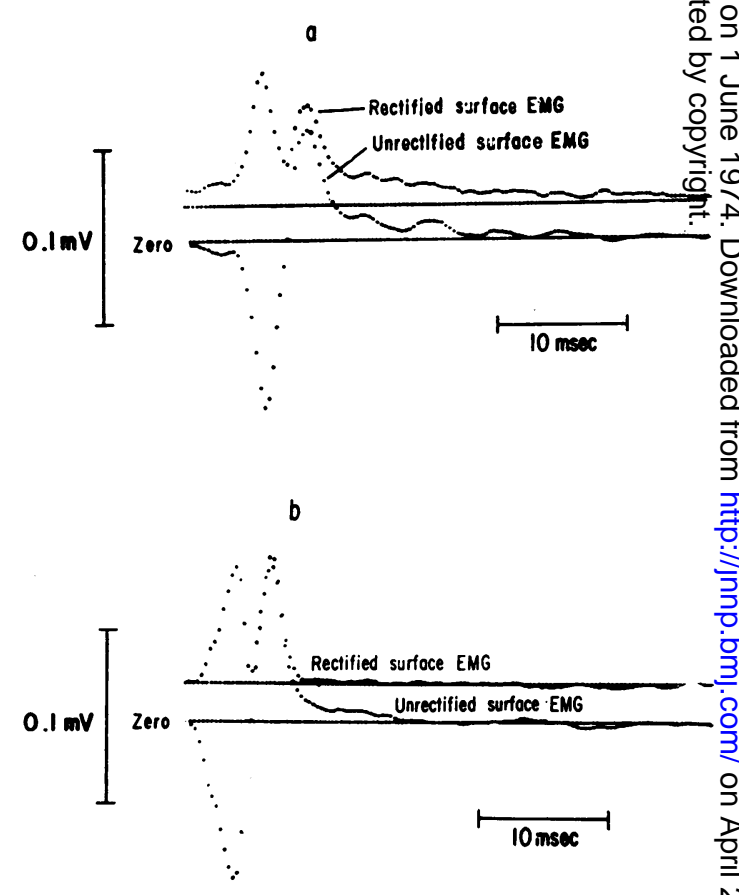

FIG. 4. Experimental traces of an averaged rectified surface EMG superimposed on an unrectified surface ${ }^{N}$ EMG, for evaluating synchronization ratios: a: norma N hand, $\mathrm{b}$ : affected hand. Horizontal lines indicateo voltages of zero and the mean rectified surface $E M G_{6}$ 


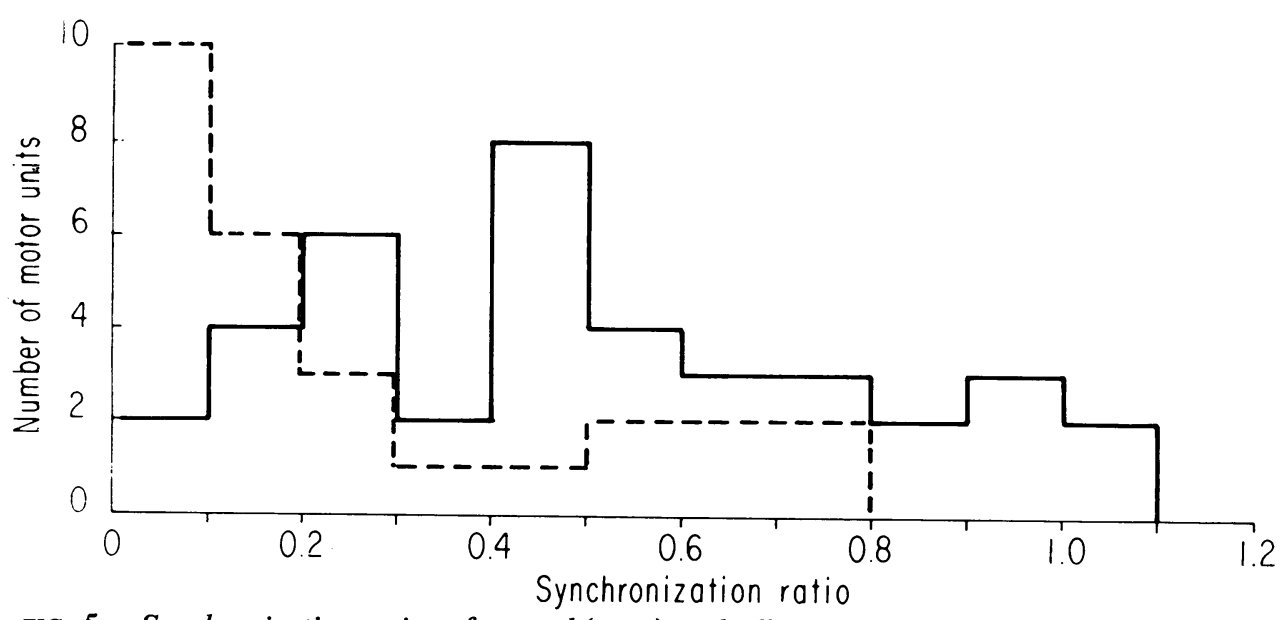

FIG. 5. Synchronization ratios of normal (-) and affected (---) hands of five patients with ulnar neuropathies who showed synchronization. The distributions of the synchronization ratios differed significantly $(P<0.01)$.
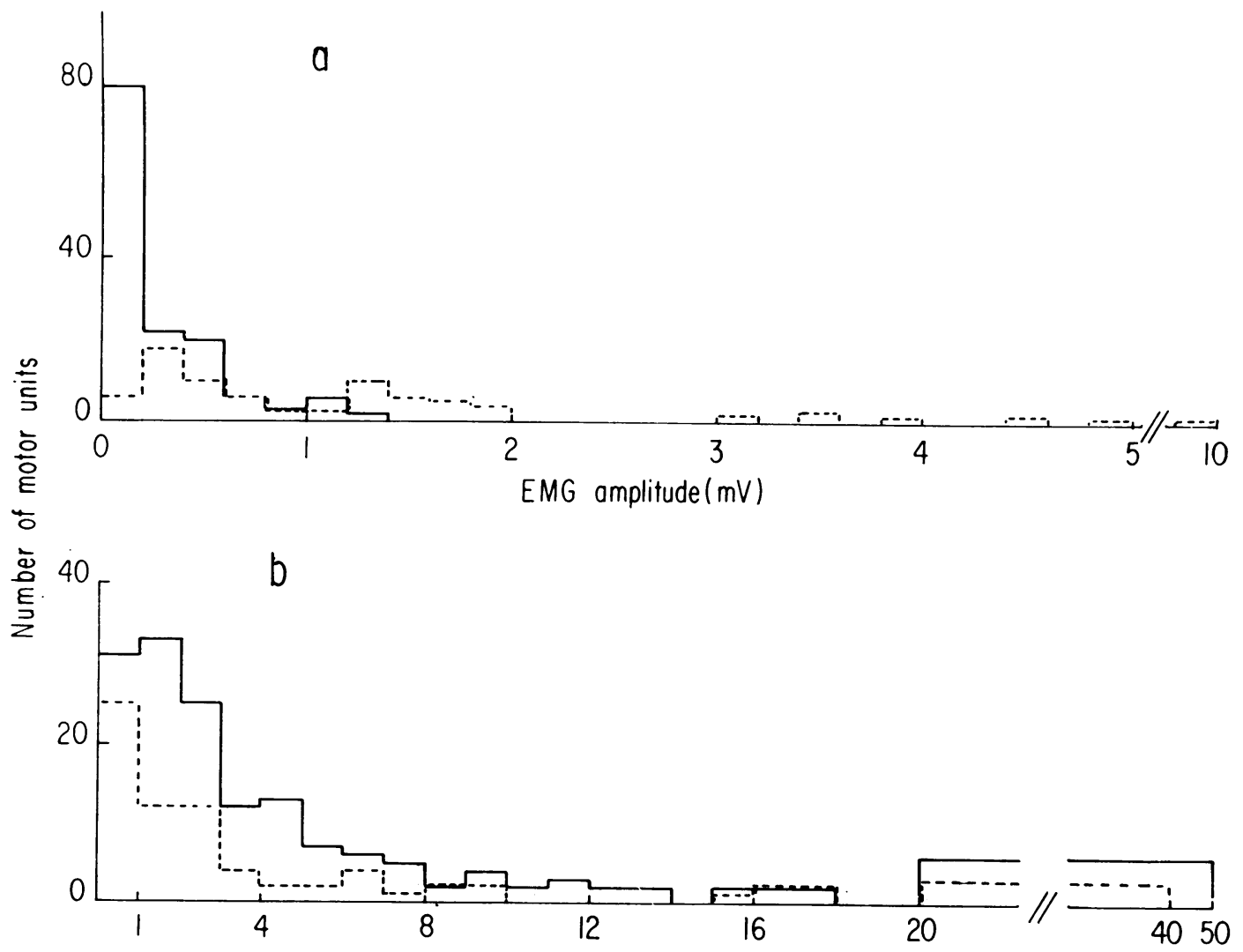

Twitch tension ( $\mathrm{g}$ )

FIG. 6. a: EMG amplitudes of motor units from 25 normal subjects (-) and eight patients with motor neurone disease (--). b: Twitch tensions of motor units from 25 normal subjects $(-)$ and eight patients with motor neurone disease (---). The distributions of the twitch tensions were not significantly different $(P>0 \cdot 8)$ but the distributions of the EMG amplitudes were significantly different $(P<0 \cdot 001)$. 
Erminio et al., 1959); the generally smaller sizes of the twitch tensions associated with these EMGs, however, suggest the involvement of other factors (see Discussion). Figure 3 shows the average of the twitch tensions recorded from the affected hands divided by the averages from the normal hands (twitch ratios). The corresponding ratios of the average EMG amplitudes of the affected and normal hands (EMG ratios $[\mathrm{A} / \mathrm{N}])$ are also given to illustrate the discrepancy between the relatively larger EMG amplitudes and the smaller twitch tensions. The distributions of the two ratios were significantly different $(\mathrm{P}<0.01)$.

Synchronization A synchronization ratio (see Methods) was calculated in all the patients with ulnar neuropathies. Figure 4 shows experimental data with a rectified surface EMG superimposed on an unrectified surface EMG for one of the patients who showed synchronized motor unit activity-(a) normal, (b) affected. Only five of the patients showed synchronized motor unit activity in their normal hand; the affected hands showed either a decrease in synchronization or a complete absence of synchronization. Figure 5 is a histogram of the synchronization ratios for the normal and affected hands of the five patients; 0.2 was taken as the lower limit for synchronized activity (see Methods).

All of the patients in our sample who showed synchronized activity were men and most worked at manual jobs which required exertion of relatively large forces with these muscles. The possible significance of this finding will be discussed later. The measured values of twitch tension were much larger than average in these patients due to the strength developed from use of the muscles, but also because the 'twitches' were generated by the somewhat synchronized activity of more than one motor unit. However, even if the data from these individuals are excluded, the differences noted in previous sections between abnormal and normal hands remain. For example, the overall mean twitcht tensions in the nine patients who showed nळे evidence of synchronization were $3.80 \pm 0.48$ (mean \pm SE of the mean) for the normal hand and $2.35 \pm 0.46 \mathrm{~g}$ for the affected hands.

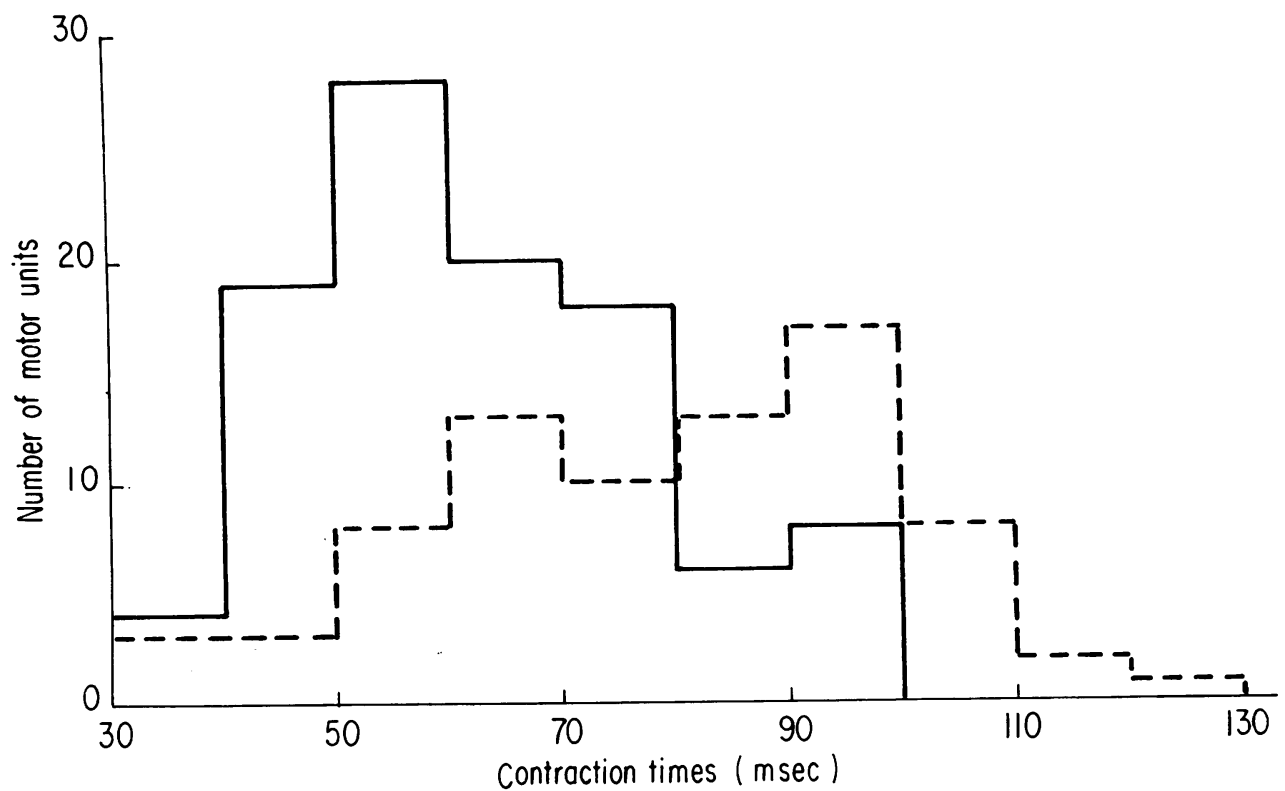

FIG. 7. Contraction times of twitches from motor units in 25 normal hands (-) and eight patients with motor neurone disease (---). The distributions of the contraction times were signifcantly different $(P<0 \cdot 01)$. 
MOTOR NEURONE DISEASE Since seven out of the eight motor neurone disease patients were bilaterally affected, the EMG amplitudes and the twitch tensions recorded were compared with corresponding values pooled from 25 normal hands. Seven of the patients had larger EMG amplitudes (2 to 10 times normal average), but the twitch tensions were within the normal range. These large EMG amplitudes suggest collateral sprouting as has been previously reported (Erminio et al., 1959). Figure 6 shows histograms of EMG amplitudes and twitch tensions of normal and motor neurone disease patients. The mean twitch tensions of the normal and affected hands were $4 \cdot 7 \pm 7 \cdot 1 \mathrm{~g}$ (mean \pm SD of an observation) and $4.5 \pm 6.9 \mathrm{~g}$ respectively; the distributions were not significantly different $(\mathrm{P}>0 \cdot 8)$. The EMG amplitudes of the normal and affected hands, $0 \cdot 22 \pm 0 \cdot 26 \mathrm{mV}$ (mean \pm SD) and $1 \cdot 29 \pm 0 \cdot 149 \mathrm{mV}$ respectively, had significantly different distributions $(P<0 \cdot 001)$. This suggests that motor units enlarged by collateral sprouting are less efficient contractile units than units of normal physiological size.

The contraction times were longer in the affected hands (Fig. 7); the mean values of the contraction times were $78 \pm 21 \mathrm{~ms}$ in the affected hands and $61 \pm 17 \mathrm{~ms}$ in the normal hands and the distributions were significantly different $(\mathrm{P}<0.01)$.

\section{DISCUSSION}

Some quantitative data of the contractile and electrical properties of motor units, altered by neuropathies and motor neurone disease, have been presented in this study. The increased EMG amplitude among patients with ulnar neuropathies and motor neurone disease are consistent with the idea that any remaining 'healthy' motoneurones may sprout new collaterals to innervate muscle fibres that had been previously denervated (Wohlfart, 1957, 1958; Coërs and Woolf, 1959; Erminio et al., 1959). However, our experiments do not rule out the possibility that the atrophy and reduction of total muscle mass often seen in these patients reduces the shunting of the signals produced by motor units so that the signals recorded are larger. Since this increase is observed with surface as well as with needle electrodes, the decreased shunting would have to be quite widespread.

In view of the evidence in favour of collateral sprouting presented in the studies quoted above, this represents the more plausible explanation of our increased EMG amplitudes. However, if there is an increase in the number of muscle fibres per motor unit as a result of sprouting, the tendency for the twitch tensions to be smaller in ulnar neuropathies is all the more surprising. The enlarged motor unit appears to be a less efficient mechanical unit. This decreased efficiency could result from (1) a deficiency in excitationcontraction coupling; or (2) a deficiency in the coupling of the contraction to an external recording device. Using scanning electron microscopy, Sakuragawa et al. (1973) found that in the early stage of neurogenic atrophy due to peripheral neuropathy, there was a disappearance of the openings of the $\mathrm{T}$ tubules and loss of the A-I banding pattern. A number of other factors could also contribute to the first category such as (1) a decreased trophic influence of motoneurones on individual muscle fibres of the enlarged motor unit, leading for example to decreased enzymatic activity; (2) an increased susceptibility to fatigue. The experiments of Edwards and Lippold (1956), indicating that the integrated EMG required to maintain a constant force increases during fatigue, suggests that a substantial decrease takes place during fatigue in the efficiency of excitation-contraction coupling. More recent evidence (Stephens and Taylor, 1972) also implicates other factors in fatigue of normal human muscle. These experiments could be repeated with patients suffering from ulnar neuropathies to test whether the common clinical observation that the muscles of the affected hands tire more easily is due to a defect in excitation-contraction coupling.

The above arguments seem to contradict previous data obtained by McComas et al.(1971b) in partially denervated muscles using stimulation techniques (Sica and McComas, 1971). In their study single twitches of the extensor digitorum brevis in patients with motor neurone disease were larger than normal. In addition, they calculated the ratio of the maximum twitch tension to that of the maximum evoked muscle response and obtained $59 \mathrm{~g} / \mathrm{mV}$ for controls, while in muscles with more than $90 \%$ denerva- 
tion, the value was $78 \mathrm{~g} / \mathrm{mV}$. Our results, however, indicate that during a maintained voluntary contraction, the distribution of twitch tensions averaged over a period of about two minutes were within the normal range in patients with motor neurone disease. Also, the corresponding ratio of mean twitch tension to mean EMG amplitude calculated from our data gives the values $21 \mathrm{~g} / \mathrm{mV}$ and $4 \mathrm{~g} / \mathrm{mV}$ for normal subjects and motor neurone disease patients respectively. This discrepancy could arise from differences in the two muscles, but the data are consistent with the hypothesis that enlarged motor units may be particularly susceptible to fatigue. Thus a single stimulus could give a greater tension, as found by McComas et al. (1971b), but with maintained use the tension might fall to within the normal range, as indicated by our results.

The second explanation suggested above, a deficiency in the coupling of the internal contraction to an external recording device, could result from fibrosis within the muscle. Fibrosis might also slow the contraction and relaxation times as observed experimentally. A slowing of these times might also result from decreased excitation-contraction coupling (Mannard and Stein, 1973), and it is likely that several of these factors contribute to making the enlarged motor units in neuromuscular disease more sluggish and less efficient.

Only five of the patients showed synchronized motor unit activity in their normal hand; the affected hands showed either a decrease in synchronization, or a complete absence of synchronization. The fact that most of the five subjects hold jobs that involve a continuous exertion of large, brief forces might be significant in explaining synchronization. This result suggests that synchronization can be developed as a result of regular, near-maximal voluntary excitation of motor units. On the other hand, the decrease in or absence of synchronization in the affected hands, which were used less during periods of pain and weakness, would indicate that disuse is an important contributory factor towards desynchronization. More evidence is obviously needed to substantiate this hypothesis.

We acknowledge the help and cooperation given by Dr. J. B. Redford, Director of Rehabilitation
Medicine, University of Alberta, in initiating this $\stackrel{\mathbb{C}}{\triangle}$ study. This study was supported by grants from the Medical Research Council of Canada and the Muscular Dystrophy Association of Canada to R. B. Stein.

\section{REFERENCES}

Buchthal, F., and Olsen, P. Z. (1970). Electromyography and muscle biopsy in infantile spinal muscular atrophy. Brain, 93, 15-30.

Coërs, C., and Woolf, A. L. (1959). The Innervation of Muscle. Blackwell: Oxford.

Edwards, R. G., and Lippold, O. C. J. (1956). The relation $\overrightarrow{\mathbb{D}}$ between force and integrated electrical activity in fatigued muscle. Journal of Physiology, 132, 677-681.

Erminio, F., Buchthal, F., and Rosenfalck, P. (1959). Motor $\overrightarrow{0}$ unit territory and muscle fiber concentration in paresis due to peripheral nerve injury and anterior horn cell involve- $\vec{\omega}$ ment. Neurology (Minneap.), 9, 657-671.

Fisz, M. (1963). Probability Theory and Mathematical Statistics. 3rd edn. Wiley: New York.

McComas, A. J., Fawcett, P. R. W., Campbell, M. J., and $\dot{\omega}$ Sica, R. E. P. (1971a). Electrophysiological estimation $\underset{\boldsymbol{d}}{ } \cdot \vec{\sigma}$ the number of motor units within a human muscle. Journ $l$ ') of Neurology, Neurosurgery, and Psychiatry, 34, 121-131Ф

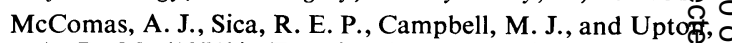
A. R. M. (1971b). Functional compensation in partiały denervated muscles. Journal of Neurology, Neurosurge and Psychiatry, 34, 453-460.

Mannard, A., and Stein, R. B. (1973). Determination of the frequency response of isometric soleus muscle in the using random nerve stimulation. Journal of Physiology, 228; 275-296.

Milner-Brown, H. S., Stein, R. B., and Yemm, R. (1973a). The contractile properties of human motor units during voluntary isometric contractions. Journal of Physiology, 228, 285-306.

Milner-Brown, H. S., Stein, R. B., and Yemm, R. (1973b). The orderly recruitment of human motor units during $\mathbb{D}$ voluntary isometric contractions. Journal of Physiology, 230, 359-370.

Payan, J. (1970). Anterior transposition of the ulnar nerve: an electrophysiological study. Journal of Neurology, Neurosurgery, and Psychiatry, 33, 157-165.

Sakuragawa, N., Sato, T., and Tsubaki, T. (1973). Scanning electron microscopic study of skeletal muscle. Archives of Neurology (Chic.), 28, 247-251.

Sica, R. E. P., and McComas, A. J. (1971). Fast and slow twitch units in a human muscle. Journal of Neurology, Neurosurgery, and Psychiatry, 34, 113-120.

Stein, R. B., French, A. S., Mannard, A., and Yemm, R. (1972). New methods for analysing motor function in man and animals. Brain Research, 40, 187-192.

Stephens, J. A., and Taylor, A. (1972). Fatigue of maintained $I$ voluntary muscle contraction in man. Journal of Physiology, 을 220, 1-18.

Wohlfart, G. (1957). Collateral regeneration from residual motor nerve fibres in amyotrophic lateral sclerosis. N Neurology (Minneap.), 7, 124-134.

Wohlfart, G. (1958). Collateral regeneration in partially $\omega$ denervated muscles. Neurology (Minneap.), 8, 175-180. 